\title{
Global stability of an SIS epidemic model with feedback mechanism on networks
}

\author{
Xiaodan Wei ${ }^{1,2^{*}}$, Gaochao Xu' and Wenshu Zhou ${ }^{3}$
}

\section{"Correspondence:}

weixiaodancat@126.com

${ }^{1}$ College of Computer Science and

Technology, Jilin University,

Changchun, China

${ }^{2}$ College of Computer Science,

Dalian Nationalities University,

Dalian, China

Full list of author information is

available at the end of the article

\section{空 Springer}

\begin{abstract}
We study the global stability of endemic equilibrium of an SIS epidemic model with feedback mechanism on networks. The model was proposed by J. Zhang and J. Sun (Physica A 394:24-32, 2014), who obtained the local asymptotic stability of endemic equilibrium. Our main purpose is to show that if the feedback parameter is sufficiently large or if the basic reproductive number belongs to the interval $(1,2]$, then the endemic equilibrium is globally asymptotically stable. We also present numerical simulations to illustrate the theoretical results.
\end{abstract}

Keywords: Epidemic model; Complex network; Feedback mechanism; Global stability

\section{Introduction}

In recent years, the spreading dynamics of epidemic models on complex networks have been extensively studied by many researchers [2-13]. In the famous works [2, 3], PastorSatorras and Vespignani established a dynamical mean-field equation based on the SIS model and showed that the finite-size effects induce an epidemic threshold, which approaches zero at increasing sizes, so that if the effective spreading rate is above this threshold, then the infection spreads and becomes endemic. For such significant finding, until recently, a mathematically rigorous proof was given by Wang and Dai [14]. Stability analysis is an important tool to understand the spreading dynamics of epidemic models on complex networks. Recently, there have been many studies concerning the stability and asymptotic behavior of epidemic models on complex networks [15-23].

We note that in most of the models mentioned, the initiative response of people is not considered when epidemic diseases prevail. In fact, as soon as an epidemic outbreaks, people are more cautious and reduce contacts with other people consciously. Clearly, the feedback mechanism can affect the contacts among people. To investigate the efficiency of feedback mechanism, new models based on the SIS model were established [1, 24-26]. Under the assumption that all individuals understand the instant situation of the propagation and can estimate the possibility of contacting with infected individuals, Zhang and Sun [1] proposed the following model with a feedback mechanism:

$$
\left\{\begin{array}{l}
\frac{d S_{k}(t)}{d t}=b\left(S_{k}(t)+I_{k}(t)\right)-d S_{k}(t)-\lambda k S_{k}(t)(1-\alpha \Theta(t)) \Theta(t)+\gamma I_{k}(t), \\
\frac{d I_{k}(t)}{d t}=\lambda k S_{k}(t)(1-\alpha \Theta(t)) \Theta(t)-(\gamma+d) I_{k}(t), \quad k=1,2, \ldots, n,
\end{array}\right.
$$

(C) The Author(s) 2018. This article is distributed under the terms of the Creative Commons Attribution 4.0 International License (http://creativecommons.org/licenses/by/4.0/), which permits unrestricted use, distribution, and reproduction in any medium, provided you give appropriate credit to the original author(s) and the source, provide a link to the Creative Commons license, and indicate if changes were made. 
where $S_{k}(t)$ and $I_{k}(t)$ represent the relative densities of susceptible and infected nodes with given degree $k$, respectively, $n$ is the maximum degree of all nodes, $\Theta(t)$ is the probability that any given link pointing to an infected individual and has the form $\Theta(t)=\langle k\rangle^{-1} \sum_{k=1}^{n} k P(k) I_{k}(t)$, where $P(k)$ is the probability that a node has degree $k$ with $\langle k\rangle=\sum_{k=1}^{n} k P(k), b$ is the natural birth rate, $d$ is the death rate, $\lambda$ is the transmission rate, $\gamma$ is recovery rate, and the parameter $\alpha>0$ is called the "fear factor." The term $\lambda k S_{k}(t)(1-\alpha \Theta(t)) \Theta(t)$ represents the proportion of individuals that acquire infection and become infected individuals. This means that, on one hand, the higher the fear degree of people to the epidemic disease, the lower the spreading speed; on the other hand, when $\Theta$ is larger than a certain threshold, the speed of the propagation decreases based on the feedback, which is consistent with the actual prevalence law of infectious diseases.

Like in [1], we assume that $b=d$ (i.e. the birth rate equals the death rate), and the initial conditions of system (1.1) satisfy

$$
0 \leq S_{k}(0), I_{k}(0) \leq 1, \quad S_{k}(0)+I_{k}(0)=1, \quad k=1,2, \ldots, n .
$$

Thus $S_{k}(t)+I_{k}(t) \equiv 1$. This leads to the following system:

$$
\left\{\begin{array}{l}
\frac{d S_{k}(t)}{d t}=b-b S_{k}(t)-\lambda k S_{k}(t)(1-\alpha \Theta(t)) \Theta(t)+\gamma I_{k}(t), \\
\frac{d I_{k}(t)}{d t}=\lambda k S_{k}(t)(1-\alpha \Theta(t)) \Theta(t)-(\gamma+b) I_{k}(t), \quad k=1,2, \ldots, n .
\end{array}\right.
$$

The second equation of (1.3) can be written as

$$
\frac{d I_{k}(t)}{d t}=\lambda k\left(1-I_{k}(t)\right)(1-\alpha \Theta(t)) \Theta(t)-(\gamma+b) I_{k}(t), \quad k=1,2, \ldots, n .
$$

They obtained the basic reproductive number $R^{0}=\frac{\lambda\left\langle k^{2}\right\rangle}{(\gamma+b)\langle k\rangle}$, where $\left\langle k^{2}\right\rangle=\sum_{k=1}^{n} k^{2} P(k)$, and proved that if $R^{0}<1$, then the disease-free equilibrium is globally asymptotically stable, and if $R^{0}>1$, then there exists a unique positive equilibrium $E^{*}\left(S_{1}^{*}, I_{1}^{*}, S_{2}^{*}, I_{2}^{*}, \ldots, S_{n}^{*}, I_{n}^{*}\right)$ determined by

$$
\left\{\begin{array}{l}
I_{k}^{*}=\frac{\lambda k\left(1-\alpha \Theta^{*}\right) \Theta^{*}}{\gamma+b+\lambda k\left(1-\alpha \Theta^{*}\right) \Theta^{*}}, \\
S_{k}^{*}=\frac{\gamma+b}{\gamma+b+\lambda k\left(1-\alpha \Theta^{*}\right) \Theta^{*}}, \quad k=1,2, \ldots, n,
\end{array}\right.
$$

where $\Theta^{*}=\langle k\rangle^{-1} \sum_{j=1}^{n} j P(j) I_{j}^{*}$ is the unique positive root of the equation

$$
f(\Theta):=1-\frac{1}{\langle k\rangle} \sum_{k=1}^{n} \frac{\lambda k^{2} P(k)(1-\alpha \Theta)}{b+\gamma+\lambda k(1-\alpha \Theta) \Theta}=0 .
$$

Moreover, $E^{*}$ is locally asymptotically stable. It should be pointed out that in the special case $\alpha=0$, which corresponds to the original model by Pastor-Satorras and Vespignani $[2,3]$, the global stability of endemic equilibrium was shown in [27]. As far as we know, however, the global asymptotic behavior of the endemic equilibrium remained unsolved for $\alpha>0$. In the present paper, we prove that the endemic equilibrium of system (1.3) is globally asymptotically stable if $\alpha \geq \frac{\lambda n}{4(b+\gamma)}\left(\frac{R^{0}-1}{R^{0}}\right)^{2}$ or if $R^{0} \in(1,2]$ and $\alpha>0$. 
We will give a remark on $\Theta^{*}$, which plays an important role in proving the following Theorems 3.1.

Remark 1.1 Following the arguments in [1, Section 2], we can see that if $R^{0}>1$, then $\Theta^{*}<$ $\alpha^{-1}$. In fact, if $\left\{\left(S_{k}^{*}, I_{k}^{*}\right)\right\}_{k=1}^{n}$ is a positive equilibrium of system (1.3), it must satisfy (1.5). It follows from $0<I_{k}^{*} S_{k}^{*}=\frac{(\gamma+b) \lambda k\left(1-\alpha \Theta^{*}\right) \Theta^{*}}{\left[\gamma+b+\lambda k\left(1-\alpha \Theta^{*}\right) \Theta^{*}\right]^{2}}$ that $\Theta^{*}<\alpha^{-1}$. On the other hand, since $f\left(\alpha^{-1}\right)=$ $1>0$ and $f(0)=1-R^{0}<0$, we find that $\Theta^{*}$ is indeed the unique root of the equation $f(\Theta)=0$ in $\left(0, \alpha^{-1}\right)$ because $f^{\prime}(\Theta)>0$ in $\left(0, \alpha^{-1}\right)$.

We further claim that $\Theta^{*} \leq \frac{R^{0}-1}{\alpha R^{0}}$ if $R^{0}>1$. By (1.5) we have $(b+\gamma) I_{k}^{*}=\lambda k\left(1-I_{k}^{*}\right)(1-$ $\left.\alpha \Theta^{*}\right) \Theta^{*} \leq \lambda k\left(1-\alpha \Theta^{*}\right) \Theta^{*}$, so $b+\gamma \leq \frac{\lambda\left\langle k^{2}\right\rangle}{\langle k\rangle}\left(1-\alpha \Theta^{*}\right)$, which implies the claim.

This paper is organized as follows. In Section 2, we show that the disease is permanent if $R^{0}>1$. In Section 3, we prove the global asymptotic stability of the endemic equilibrium for sufficiently large $\alpha$ by using a Lyapunov function. In Section 4 , we show the global asymptotic stability of the endemic equilibrium for $R^{0} \in(1,2]$ and $\alpha>0$ by the monotone iterative technique. In Section 5, we present numerical simulations. In Section 6, we give our conclusions.

\section{Permanence of disease}

In this section, we state our main results as follows.

Theorem 2.1 Let $R^{0}>1$. Assume that $\left\{\left(S_{k}, I_{k}\right)\right\}_{k=1}^{n}$ is a nonnegative solution of system (1.3) with the initial conditions (1.2) and $\Theta(0)>0$. Then $\Theta(t)>0$ for all $t \geq 0$, and

$$
\begin{aligned}
& \liminf _{t \rightarrow \infty} \Theta(t) \geq \frac{\left(R^{0}-1\right)}{\alpha R^{0}+\lambda n(\gamma+b)^{-1}}=: \theta_{\alpha}, \\
& \limsup _{t \rightarrow \infty} \Theta(t) \leq \frac{\left(R^{0}-1\right)}{\alpha R^{0}},
\end{aligned}
$$

and

$$
\begin{aligned}
& \liminf _{t \rightarrow \infty} I_{k}(t) \geq \frac{\lambda k \theta_{\alpha}}{(\gamma+b) R^{0}+\lambda k \theta_{\alpha}}, \\
& \limsup _{t \rightarrow \infty} I_{k}(t) \leq \frac{\lambda k}{4 \alpha(\gamma+b)+\lambda k} .
\end{aligned}
$$

Proof From (1.4) we have

$$
\begin{aligned}
\frac{d \Theta}{d t} & =\frac{\lambda}{\langle k\rangle} \sum_{j=1}^{n} j^{2} P(j)\left(1-I_{j}\right)(1-\alpha \Theta) \Theta-(\gamma+b) \Theta \\
& =\left(\frac{\lambda\left\langle k^{2}\right\rangle}{\langle k\rangle}-\gamma-b\right) \Theta-\frac{\alpha \lambda\left\langle k^{2}\right\rangle}{\langle k\rangle} \Theta^{2}-\frac{\lambda}{\langle k\rangle} \sum_{j=1}^{n} j^{2} P(j) I_{j} \Theta+\frac{\alpha \lambda}{\langle k\rangle} \sum_{j=1}^{n} j^{2} P(j) I_{j} \Theta^{2} \\
& =: Y(t) \Theta(t),
\end{aligned}
$$


which gives that $\Theta(t)=\Theta(0) \exp \left\{\int_{0}^{t} Y(s) d s\right\}>0$ for all $t \geq 0$. By [1, Theorem 2.1$], 0 \leq I_{k} \leq 1$ for $k=1,2, \ldots, n$, thus we deduce from (2.3) that

$$
\begin{aligned}
\frac{d \Theta}{d t} & \geq\left(\frac{\lambda\left\langle k^{2}\right\rangle}{\langle k\rangle}-\gamma-b\right) \Theta-\frac{\alpha \lambda\left\langle k^{2}\right\rangle}{\langle k\rangle} \Theta^{2}-\frac{\lambda}{\langle k\rangle} \sum_{j=1}^{n} j^{2} P(j) I_{j} \Theta \\
& \geq\left(\frac{\lambda\left\langle k^{2}\right\rangle}{\langle k\rangle}-\gamma-b\right) \Theta-\frac{\alpha \lambda\left\langle k^{2}\right\rangle}{\langle k\rangle} \Theta^{2}-\lambda n \Theta^{2} \\
& =(\gamma+b)\left\{\left(R^{0}-1\right) \Theta-\left[\alpha R^{0}+\lambda n(\gamma+b)^{-1}\right] \Theta^{2}\right\},
\end{aligned}
$$

which implies $\liminf _{t \rightarrow \infty} \Theta(t) \geq \theta_{\alpha}$.

We further show that $\lim \sup _{t \rightarrow \infty} \Theta(t) \leq \frac{\left(R^{0}-1\right)}{\alpha R^{0}}$. Since $0 \leq I_{k} \leq 1$ for $k=1,2, \ldots, n$, we can deduce from (2.3) that $\frac{d^{2} \Theta(t)}{d t^{2}}$ is bounded in $(0, \infty)$. By Corollary $C$ in the Appendix, there is a sequence $\left\{t_{m}\right\}$, increasing to infinity, such that

$$
\theta_{\alpha} \leq \lim _{m \rightarrow \infty} \Theta\left(t_{m}\right)=\limsup _{t \rightarrow \infty} \Theta(t)=: \theta, \quad \lim _{m \rightarrow \infty} \Theta^{\prime}\left(t_{m}\right)=0 .
$$

On the other hand, since $\left\{I_{k}\left(t_{m}\right)\right\}_{m=1}^{\infty} \in[0,1]$ for every $k$, there is a subsequence of $\left\{t_{m}\right\}_{m=1}^{\infty}$, denoted by itself, and some constants $i_{k} \in[0,1]$ for $k=1,2, \ldots, n$ such that $I_{k}\left(t_{m}\right) \rightarrow i_{k}$ $(m \rightarrow \infty)$. Passing to the limit as $m \rightarrow \infty$ in (2.3) at $t=t_{m}$ yields

$$
(\gamma+b) \theta=\frac{\lambda}{\langle k\rangle} \sum_{j=1}^{n} j^{2} P(j)\left(1-i_{k}\right)(1-\alpha \theta) \theta \leq \frac{\lambda\left\langle k^{2}\right\rangle}{\langle k\rangle}(1-\alpha \theta) \theta .
$$

This implies the required result.

Finally, we prove (2.2). Note that $\frac{d^{2} I_{k}(t)}{d t^{2}}$ is bounded in $(0, \infty)$ for every $k$. Based on Corollary $C$ in the Appendix and (2.1), we can derive by an argument similar to the previous one that, for any fixed $k \in\{1,2, \ldots, n\}$, there are two sequences $\left\{t_{m}^{(k)}\right\}$ and $\left\{s_{m}^{(k)}\right\}$, increasing to infinity, and some constants $\bar{\theta}_{k}, \underline{\theta}_{k} \in\left[\theta_{\alpha}, \frac{\left(R^{0}-1\right)}{\alpha R^{0}}\right]$ such that

$$
\begin{array}{ll}
\lim _{m \rightarrow \infty} I_{k}\left(t_{m}^{(k)}\right)=\limsup _{t \rightarrow \infty} I_{k}=: \bar{i}_{k} \in[0,1], & \lim _{m \rightarrow \infty} I_{k}^{\prime}\left(t_{m}^{(k)}\right)=0, \\
\lim _{m \rightarrow \infty} I_{k}\left(s_{m}^{(k)}\right)=\liminf _{t \rightarrow \infty} I_{k}=: \underline{i}_{k} \in[0,1], \quad \lim _{m \rightarrow \infty} I_{k}^{\prime}\left(s_{m}^{(k)}\right)=0, \\
\lim _{m \rightarrow \infty} \Theta\left(t_{m}^{(k)}\right)=\bar{\theta}_{k}, \quad \lim _{m \rightarrow \infty} \Theta\left(s_{m}^{(k)}\right)=\underline{\theta}_{k} . &
\end{array}
$$

Passing to the limits as $m \rightarrow \infty$ in (1.4) at $t=t_{m}^{(k)}$ and $t=s_{m}^{(k)}$, respectively, we obtain

$$
\begin{aligned}
& 0=\lambda k\left(1-\bar{i}_{k}\right)\left(1-\alpha \bar{\theta}_{k}\right) \bar{\theta}_{k}-(\gamma+b) \bar{i}_{k}, \\
& 0=\lambda k\left(1-\underline{i}_{k}\right)\left(1-\alpha \underline{\theta}_{k}\right) \underline{\theta}_{k}-(\gamma+b) \underline{i}_{k},
\end{aligned}
$$

and therefore

$$
\bar{i}_{k}=\frac{\lambda k\left(1-\alpha \bar{\theta}_{k}\right) \bar{\theta}_{k}}{\gamma+b+\lambda k\left(1-\alpha \bar{\theta}_{k}\right) \bar{\theta}_{k}}, \quad \underline{i}_{k}=\frac{\lambda k\left(1-\alpha \underline{\theta}_{k}\right) \underline{\theta}_{k}}{\gamma+b+\lambda k\left(1-\alpha \underline{\theta}_{k}\right) \underline{\theta}_{k}} .
$$


By the inequality $x(1-\alpha x) \leq \frac{1}{4 \alpha}$ for $x \in \mathbb{R}$ we have

$$
\bar{i}_{k} \leq \frac{\frac{\lambda k}{4 \alpha}}{\gamma+b+\frac{\lambda k}{4 \alpha}}=\frac{\lambda k}{4 \alpha(\gamma+b)+\lambda k}, \quad k=1,2, \ldots, n .
$$

Since $\theta_{\alpha} \leq \underline{\theta}_{k} \leq \frac{\left(R^{0}-1\right)}{\alpha R^{0}}$, we have $\underline{i}_{k} \geq \frac{\frac{\lambda k \theta_{\alpha}}{R^{0}}}{b+\gamma+\frac{\lambda k \theta_{\alpha}}{R^{0}}}$. Thus, the proof is completed.

Remark 2.1 When $R^{0}=1$ (i.e., $\gamma+b=\frac{\lambda\left\langle k^{2}\right\rangle}{\langle k\rangle}$ ), we can obtain (2.4) by a similar argument, where $\theta \in[0,1]$, which implies that $\limsup _{t \rightarrow \infty} \Theta=\theta=0$, so $\lim _{t \rightarrow \infty} I_{k}(t)=0$ for $k=1,2, \ldots, n$, i.e., the disease-free equilibrium is globally attractive. This can also be seen from (2.1) by letting $R^{0} \rightarrow 1^{+}$. This result is a supplement of [1, Theorem 3.1].

\section{Global stability for large $\alpha$}

The main result of this section can be stated as follows.

Theorem 3.1 Let $R^{0}>1$ and $\Theta(0)>0$. If $\alpha \geq \frac{\lambda n}{4(b+\gamma)}\left(\frac{R^{0}-1}{R^{0}}\right)^{2}$, then the endemic equilibrium $E^{*}$ of system (1.3) is globally asymptotically stable, that is, the disease becomes endemic.

Proof According to [1, Theorem 3.2], the endemic equilibrium $E^{*}$ is locally asymptotically stable. To prove the theorem, it suffices to show that $E^{*}$ is globally attractive.

Let us consider a nonnegative solution $\left\{\left(S_{k}, I_{k}\right)\right\}_{k=1}^{n}$ and define

$$
V(t)=\frac{1}{2} \sum_{k=1}^{n} a_{k}\left(S_{k}(t)-S_{k}^{*}\right)^{2}+\Theta(t)-\Theta^{*}-\Theta^{*} \ln \frac{\Theta(t)}{\Theta^{*}}
$$

where $a_{k}=\frac{k P(k)}{\langle k\rangle S_{k}^{*}}$. Calculating the derivative of $V(t)$ along the solution, we have

$$
\frac{d V}{d t}=\sum_{k=1}^{n} a_{k}\left(S_{k}-S_{k}^{*}\right) \frac{d S_{k}}{d t}+\frac{\Theta-\Theta^{*}}{\Theta} \frac{d \Theta}{d t}=: V_{1}+V_{2}
$$

Using the first equation of (1.3) and the identity $\gamma+b=(\gamma+b) S_{k}^{*}+\lambda k S_{k}^{*}\left(1-\alpha \Theta^{*}\right) \Theta^{*}$, we have

$$
V_{1}=\sum_{k=1}^{n} a_{k}\left(S_{k}-S_{k}^{*}\right)\left\{(\gamma+b)\left(S_{k}^{*}-S_{k}\right)+\lambda k\left[S_{k}^{*}\left(1-\alpha \Theta^{*}\right) \Theta^{*}-S_{k}(1-\alpha \Theta) \Theta\right]\right\} .
$$

Note that

$$
\begin{aligned}
& S_{k}^{*}\left(1-\alpha \Theta^{*}\right) \Theta^{*}-S_{k}(1-\alpha \Theta) \Theta \\
& \quad=(1-\alpha \Theta) \Theta\left(S_{k}^{*}-S_{k}\right)-S_{k}^{*}\left(1-2 \alpha \Theta^{*}\right)\left(\Theta-\Theta^{*}\right)+\alpha S_{k}^{*}\left(\Theta-\Theta^{*}\right)^{2} .
\end{aligned}
$$

Substituting this into (3.1) yields

$$
\begin{aligned}
V_{1}= & -\sum_{k=1}^{n}(\gamma+b) a_{k}\left(S_{k}-S_{k}^{*}\right)^{2}-(1-\alpha \Theta) \Theta \sum_{k=1}^{n} \lambda k a_{k}\left(S_{k}-S_{k}^{*}\right)^{2} \\
& -\sum_{k=1}^{n} \lambda k a_{k} S_{k}^{*}\left(1-2 \alpha \Theta^{*}\right)\left(S_{k}-S_{k}^{*}\right)\left(\Theta-\Theta^{*}\right)+\sum_{k=1}^{n} \alpha \lambda k a_{k} S_{k}^{*}\left(S_{k}-S_{k}^{*}\right)\left(\Theta^{*}-\Theta\right)^{2}
\end{aligned}
$$




$$
\begin{aligned}
= & -\sum_{k=1}^{n}(\gamma+b) a_{k}\left(S_{k}-S_{k}^{*}\right)^{2}-(1-\alpha \Theta) \Theta \sum_{k=1}^{n} \lambda k a_{k}\left(S_{k}-S_{k}^{*}\right)^{2} \\
& +\sum_{k=1}^{n} \alpha \lambda k a_{k} S_{k}^{*} \Theta^{*}\left(S_{k}-S_{k}^{*}\right)\left(\Theta-\Theta^{*}\right)-\sum_{k=1}^{n} \alpha \lambda k a_{k}\left(S_{k}^{*}\right)^{2}\left(\Theta-\Theta^{*}\right)^{2} \\
& -\sum_{k=1}^{n} \lambda k a_{k} S_{k}^{*}\left[\left(1-\alpha \Theta^{*}\right)\left(S_{k}-S_{k}^{*}\right)\left(\Theta-\Theta^{*}\right)-\alpha S_{k}\left(\Theta-\Theta^{*}\right)^{2}\right] .
\end{aligned}
$$

Using (2.3) and the identity $\gamma+b=\frac{\lambda}{\langle k\rangle} \sum_{k=1}^{n} k^{2} P(k) S_{k}^{*}\left(1-\alpha \Theta^{*}\right)$, we have

$$
\begin{aligned}
V_{2} & =\left(\Theta-\Theta^{*}\right)\left[-(\gamma+b)+\frac{\lambda}{\langle k\rangle} \sum_{k=1}^{n} k^{2} P(k) S_{k}(1-\alpha \Theta)\right] \\
& =\left(\Theta-\Theta^{*}\right) \frac{\lambda}{\langle k\rangle} \sum_{k=1}^{n} k^{2} P(k)\left[S_{k}(1-\alpha \Theta)-S_{k}^{*}\left(1-\alpha \Theta^{*}\right)\right] \\
& =\frac{\lambda}{\langle k\rangle} \sum_{k=1}^{n} k^{2} P(k)\left[\left(1-\alpha \Theta^{*}\right)\left(S_{k}-S_{k}^{*}\right)\left(\Theta-\Theta^{*}\right)-\alpha S_{k}\left(\Theta-\Theta^{*}\right)^{2}\right] .
\end{aligned}
$$

Adding (3.2) and (3.3) and noticing that $a_{k}=\frac{k P(k)}{|k\rangle S_{k}^{*}}$, we obtain

$$
\begin{aligned}
\frac{d V}{d t}= & -\sum_{k=1}^{n}(\gamma+b) a_{k}\left(S_{k}-S_{k}^{*}\right)^{2}-(1-\alpha \Theta) \Theta \sum_{k=1}^{n} \lambda k a_{k}\left(S_{k}-S_{k}^{*}\right)^{2} \\
& +\sum_{k=1}^{n} \alpha \lambda k a_{k} S_{k}^{*} \Theta^{*}\left(S_{k}-S_{k}^{*}\right)\left(\Theta-\Theta^{*}\right)-\sum_{k=1}^{n} \alpha \lambda k a_{k}\left(S_{k}^{*}\right)^{2}\left(\Theta-\Theta^{*}\right)^{2} \\
= & -(1-\alpha \Theta) \Theta \sum_{k=1}^{n} \lambda k a_{k}\left(S_{k}-S_{k}^{*}\right)^{2} \\
& -\sum_{k=1}^{n} a_{k}\left[(\gamma+b) Z_{k}^{2}-\alpha \lambda k S_{k}^{*} \Theta^{*} Z_{k} X+\alpha \lambda k\left(S_{k}^{*}\right)^{2} X^{2}\right]
\end{aligned}
$$

where $Z_{k}=S_{k}-S_{k}^{*}$ and $X=\Theta-\Theta^{*}$. Note that $\Theta^{*} \leq \frac{R^{0}-1}{\alpha R^{0}}$ (see Remark 1.1). Then if $\alpha \geq$ $\frac{\lambda n}{4(b+\gamma)}\left(\frac{R^{0}-1}{R^{0}}\right)^{2}$, then we have

$$
\begin{aligned}
\Delta & :=\left(\alpha \lambda k S_{k}^{*} \Theta^{*}\right)^{2}-4(\gamma+b) \alpha \lambda k\left(S_{k}^{*}\right)^{2} \\
& \leq\left(\lambda k S_{k}^{*}\right)^{2}\left(\frac{R^{0}-1}{R^{0}}\right)^{2}-4(\gamma+b) \alpha \lambda k\left(S_{k}^{*}\right)^{2} \\
& \leq \lambda k\left(S_{k}^{*}\right)^{2}\left[\lambda n\left(\frac{R^{0}-1}{R^{0}}\right)^{2}-4 \alpha(\gamma+b)\right] \leq 0 .
\end{aligned}
$$

This implies that

$$
(\gamma+b) Z_{k}^{2}-\alpha \lambda k S_{k}^{*} \Theta^{*} Z_{k} X+\alpha \lambda k\left(S_{k}^{*}\right)^{2} X^{2} \geq 0, \quad k=1,2, \ldots, n .
$$


It follows from (3.4) that

$$
\frac{d V}{d t} \leq-(1-\alpha \Theta) \Theta \sum_{k=1}^{n} \lambda k a_{k}\left(S_{k}-S_{k}^{*}\right)^{2}, \quad \forall t>0
$$

By (2.1), there exists some $T>0$ such that $\Theta(t)<\alpha^{-1}$ for all $t \geq T$. Consequently,

$$
\frac{d V}{d t} \leq-(1-\alpha \Theta) \Theta \sum_{k=1}^{n} \lambda k a_{k}\left(S_{k}-S_{k}^{*}\right)^{2} \leq 0, \quad \forall t \geq T
$$

Therefore, the only invariant set in $\left\{\frac{d V}{d t}=0, t \geq T\right\}$ is the singleton $\left\{E^{*}\right\}$. According to the LaSalle invariant principle [28], $E^{*}$ is globally attractive, so $E^{*}$ is globally asymptotically stable. The proof is completed.

\section{Global stability for $R^{0} \in(1,2]$}

Theorem 4.1 Let $R^{0}>1$ and $\Theta(0)>0$. If $R^{0} \leq 2$, then the endemic equilibrium $E^{*}$ of system (1.3) is globally asymptotically stable for any $\alpha>0$.

Proof According to [1, Theorem 3.2], the endemic equilibrium $E^{*}$ is locally asymptotically stable. To prove the theorem, it suffices to show that

$$
\lim _{t \rightarrow \infty} I_{k}(t)=I_{k}^{*}, \quad k=1,2, \ldots, n
$$

To this end, we will use an iterative technique and define the sequence $\left\{u_{k}^{(m)}\right\}_{m=1}^{\infty}$ for $k=$ $1,2, \ldots, n$ by

$$
\left\{\begin{array}{l}
u_{k}^{(1)}=\frac{\lambda k}{4 \alpha(\gamma+b)+\lambda k}<\frac{\lambda k}{4 \alpha(\gamma+b)}, \\
u_{k}^{(m+1)}=g_{k}\left(U_{m}\right), \quad U_{m}=\langle k\rangle^{-1} \sum_{j=1}^{n} j P(j) u_{j}^{(m)}, \quad m=1,2, \ldots,
\end{array}\right.
$$

where

$$
g_{k}(x)= \begin{cases}\frac{\lambda k(1-\alpha x) x}{\gamma+b+\lambda k(1-\alpha x) x}, & \forall x \in\left[0, \alpha^{-1}\right] \\ 0, & \forall x \in(-\infty, 0) \cup\left(\alpha^{-1},+\infty\right) .\end{cases}
$$

Note that, for every $k=1,2, \ldots, n$,

$$
g_{k}(x) \text { is increasing in } x \text { on }\left[0, \frac{1}{2 \alpha}\right] \text {. }
$$

Since $1<R^{0} \leq 2$, we obtain

$$
0<U_{1}=\langle k\rangle^{-1} \sum_{j=1}^{n} j P(j) u_{j}^{(1)}<\frac{\lambda\left\langle k^{2}\right\rangle}{4 \alpha(\gamma+b)\langle k\rangle}=\frac{R^{0}}{4 \alpha} \leq \frac{1}{2 \alpha} .
$$


By (4.1) we have

$$
\begin{aligned}
0 & <u_{k}^{(2)}=g_{k}\left(U_{1}\right)=\frac{\lambda k\left(1-\alpha U_{1}\right) U_{1}}{\gamma+b+\lambda k\left(1-\alpha U_{1}\right) U_{1}} \\
& \leq \frac{\lambda k}{4 \alpha(\gamma+b)+\lambda k}=u_{k}^{(1)} \\
& <\frac{\lambda k}{4 \alpha(\gamma+b)} .
\end{aligned}
$$

This leads to

$$
0<U_{2}=\langle k\rangle^{-1} \sum_{j=1}^{n} j P(j) u_{j}^{(2)}<\frac{\lambda\left\langle k^{2}\right\rangle}{4 \alpha(\gamma+b)\langle k\rangle}=\frac{R^{0}}{4 \alpha} \leq \frac{1}{2 \alpha}
$$

and therefore $0<U_{2} \leq U_{1}<\frac{1}{2 \alpha}$ by (4.2). Thus, we have, by (4.1),

$$
\begin{aligned}
0 & <u_{k}^{(3)}=g_{k}\left(U_{2}\right)=\frac{\lambda k\left(1-\alpha U_{2}\right) U_{2}}{\gamma+b+\lambda k\left(1-\alpha U_{2}\right) U_{2}} \\
& \leq \frac{\lambda k\left(1-\alpha U_{1}\right) U_{1}}{\gamma+b+\lambda k\left(1-\alpha U_{1}\right) U_{1}}=u_{k}^{(2)}<\frac{\lambda k}{4 \alpha(\gamma+b)} .
\end{aligned}
$$

By induction we find that

$$
0<U_{m}<\frac{1}{2 \alpha}, \quad 0<u_{k}^{(m+1)} \leq u_{k}^{(m)}<\frac{\lambda k}{4 \alpha(\gamma+b)}, \quad m=1,2, \ldots
$$

and

$$
u_{k}^{(m+1)}=g_{k}\left(U_{m}\right)=\frac{\lambda k\left(1-\alpha U_{m}\right) U_{m}}{\gamma+b+\lambda k\left(1-\alpha U_{m}\right) U_{m}}, \quad m=1,2, \ldots
$$

Using (2.2) yields limsup $\sup _{t \rightarrow \infty} I_{k}(t) \leq u_{k}^{(1)}$. Thus, applying Lemma D in the Appendix repeatedly, we have

$$
\limsup _{t \rightarrow \infty} I_{k}(t) \leq u_{k}^{(m)}, \quad k=1,2, \ldots, n, m=1,2, \ldots
$$

Since by (4.3) the sequence $\left\{u_{k}^{(m)}\right\}_{m=1}^{\infty}$ is nonincreasing for every $k$, its limit exists and is denoted by $\lim _{m \rightarrow \infty} u_{k}^{(m)}=u_{k}$. Letting $m \rightarrow \infty$ in (4.4) and (4.5), respectively, we obtain

$$
\begin{aligned}
& u_{k}=\frac{\lambda k\left(1-\alpha\langle k\rangle^{-1} \sum_{j=1}^{n} j P(j) u_{j}\right)\langle k\rangle^{-1} \sum_{j=1}^{n} j P(j) u_{j}}{\gamma+b+\lambda k\left(1-\alpha\langle k\rangle^{-1} \sum_{j=1}^{n} j P(j) u_{j}\right)\langle k\rangle^{-1} \sum_{j=1}^{n} j P(j) u_{j}}, \\
& \limsup _{t \rightarrow \infty} I_{k}(t) \leq u_{k}, \quad k=1,2, \ldots, n .
\end{aligned}
$$

On the other hand, we consider the function

$$
f(x)=\frac{1}{\langle k\rangle} \sum_{j=1}^{n} \frac{\lambda j^{2} P(j) x(1-\alpha x)}{\gamma+b+\lambda j x(1-\alpha x)}-x, \quad \forall x \in\left[0, \alpha^{-1}\right) .
$$


A simple calculation gives $f^{\prime}(0)=R^{0}-1>0$, so $f(x)>0$ for each small $x>0$. Due to (2.2), we can choose $l_{k}^{(1)}$ for $k=1,2, \ldots, n$ such that

$$
0<l_{k}^{(1)}<\liminf _{t \rightarrow \infty} I_{k}(t)<\frac{1}{\alpha}, \quad f\left(\langle k\rangle^{-1} \sum_{j=1}^{n} j P(j) l_{j}^{(1)}\right)>0 .
$$

We now define the sequence $\left\{l_{k}^{(m)}\right\}_{m=1}^{\infty}$ for $k=1,2, \ldots, n$ by

$$
l_{k}^{(m+1)}=g_{k}\left(L_{m}\right), \quad L_{m}=\langle k\rangle^{-1} \sum_{j=1}^{n} j P(j) l_{j}^{(m)}, \quad m=1,2, \ldots
$$

Since $1<R^{0} \leq 2$, a similar argument gives

$$
0<l_{k}^{(m)}<\frac{\lambda k}{4 \alpha(\gamma+b)}, \quad 0<L_{m}<\frac{1}{2 \alpha}, \quad m=1,2, \ldots
$$

It follows from the definition of $g_{k}$ that

$$
l_{k}^{(m+1)}=\frac{\lambda k\left(1-\alpha L_{m}\right) L_{m}}{\gamma+b+\lambda k\left(1-\alpha L_{m}\right) L_{m}}, \quad m=1,2, \ldots
$$

Using (4.7) and applying Lemma D in the Appendix repeatedly, we have

$$
\liminf _{t \rightarrow \infty} I_{k}(t) \geq l_{k}^{(m)}, \quad k=1,2, \ldots, n, m=1,2, \ldots
$$

Since $\frac{1}{2 \alpha}>L_{2}>L_{1}>0$ by (4.7) and (4.8), we have, by (4.1),

$$
l_{k}^{(3)}=\frac{\lambda k\left(1-\alpha L_{2}\right) L_{2}}{\gamma+b+\lambda k\left(1-\alpha L_{2}\right) L_{2}} \geq \frac{\lambda k\left(1-\alpha L_{1}\right) L_{1}}{\gamma+b+\lambda k\left(1-\alpha L_{1}\right) L_{1}}=l_{k}^{(2)} .
$$

So $\frac{1}{2 \alpha}>L_{3} \geq L_{2}>0$, and thus

$$
l_{k}^{(4)}=\frac{\lambda k\left(1-\alpha L_{3}\right) L_{3}}{\gamma+b+\lambda k\left(1-\alpha L_{3}\right) L_{3}} \geq \frac{\lambda k\left(1-\alpha L_{2}\right) L_{2}}{\gamma+b+\lambda k\left(1-\alpha L_{2}\right) L_{2}}=l_{k}^{(3)} .
$$

By induction we find that the sequence $\left\{l_{k}^{(m)}\right\}_{m=2}^{\infty}$ is nondecreasing for every $k$, so its limit exists and is denoted by $\lim _{m \rightarrow \infty} l_{k}^{(m)}=l_{k}$. Letting $m \rightarrow \infty$ in (4.8) and (4.9). respectively, we obtain

$$
\begin{aligned}
& l_{k}=\frac{\lambda k\left(1-\alpha\langle k\rangle^{-1} \sum_{j=1}^{n} j P(j) l_{j}\right)\langle k\rangle^{-1} \sum_{j=1}^{n} j P(j) l_{j}}{\gamma+b+\lambda k\left(1-\alpha\langle k\rangle^{-1} \sum_{j=1}^{n} j P(j) l_{j}\right)\langle k\rangle^{-1} \sum_{j=1}^{n} j P(j) l_{j}}, \\
& \liminf _{t \rightarrow \infty} I_{k}(t) \geq l_{k}, \quad k=1,2, \ldots, n .
\end{aligned}
$$

Finally, it follows from the uniqueness of positive solutions of (1.6) that $u_{k}=l_{k}=I_{k}^{*}$, so

$$
\liminf _{t \rightarrow \infty} I_{k}(t)=\limsup _{t \rightarrow \infty} I_{k}(t)=I_{k}^{*}
$$

The proof is completed. 
Remark 4.1 According to Theorems 3.1 and 4.1, we have that if $R^{0} \in(1,2]$ and $\alpha>0$, or if $R^{0}>2$ and $\alpha \geq \alpha_{n}:=\frac{\lambda n}{4(b+\gamma)}\left(\frac{R^{0}-1}{R^{0}}\right)^{2}$, then the endemic equilibrium of (1.1) is globally asymptotically stable. On the other hand, if we consider the scale-free network (i.e., $\left.P(k) \sim k^{-c}(2<c \leq 3)\right)$ and fix the parameters $\lambda, b$, and $\gamma$, then $R^{0} \rightarrow+\infty$ and $\alpha_{n} \rightarrow+\infty$ as $n \rightarrow+\infty$. This means that when the maximum degree $n$ is sufficiently large, we have $R^{0}>2$, so the endemic equilibrium of (1.1) is globally asymptotically stable for sufficiently large $\alpha$ by Theorem 3.1 .

\section{Numerical simulations}

In this section, we present numerical simulations to illustrate the theoretical results. We consider (1.4) on a finite scale-free network with $n=500$ and $P(k)=a k^{-3}$, where the constant $a$ satisfies $\sum_{k=1}^{500} P(k)=1$. Recall that $\alpha_{n}=\frac{\lambda n}{4(b+\gamma)}\left(\frac{R^{0}-1}{R^{0}}\right)^{2}$.

In Figure 1, we fix the parameters $b=0.31, \gamma=0.41$, and $\lambda=0.26$ to plot the time evolution of $I(t)$ with different $\alpha$, where $I(t)=\sum_{k=1}^{500} P(k) I_{k}(t)$ is the density of infected nodes in the whole network. We can verify that $R^{0}=1.4930<2$. Obviously, the orbits converge to stationary levels, which illustrates the correctness of Theorem 4.1. Moreover, we can observe from the simulation that the larger the $\alpha$, the weaker the disease, which is consistent with the theoretical result (2.2).

In Figure 2, we fix the parameters $b=0.4, \gamma=0.3$, and $\lambda=0.34$ to plot the time evolution of $I(t)$ with five different initial values. We can verify that $R^{0}=2.0082>2$ and $\alpha_{500}=15.3028$. We choose $\alpha=30$ in Figure 2 (left) and $\alpha=15$ in Figure 2 (right). The
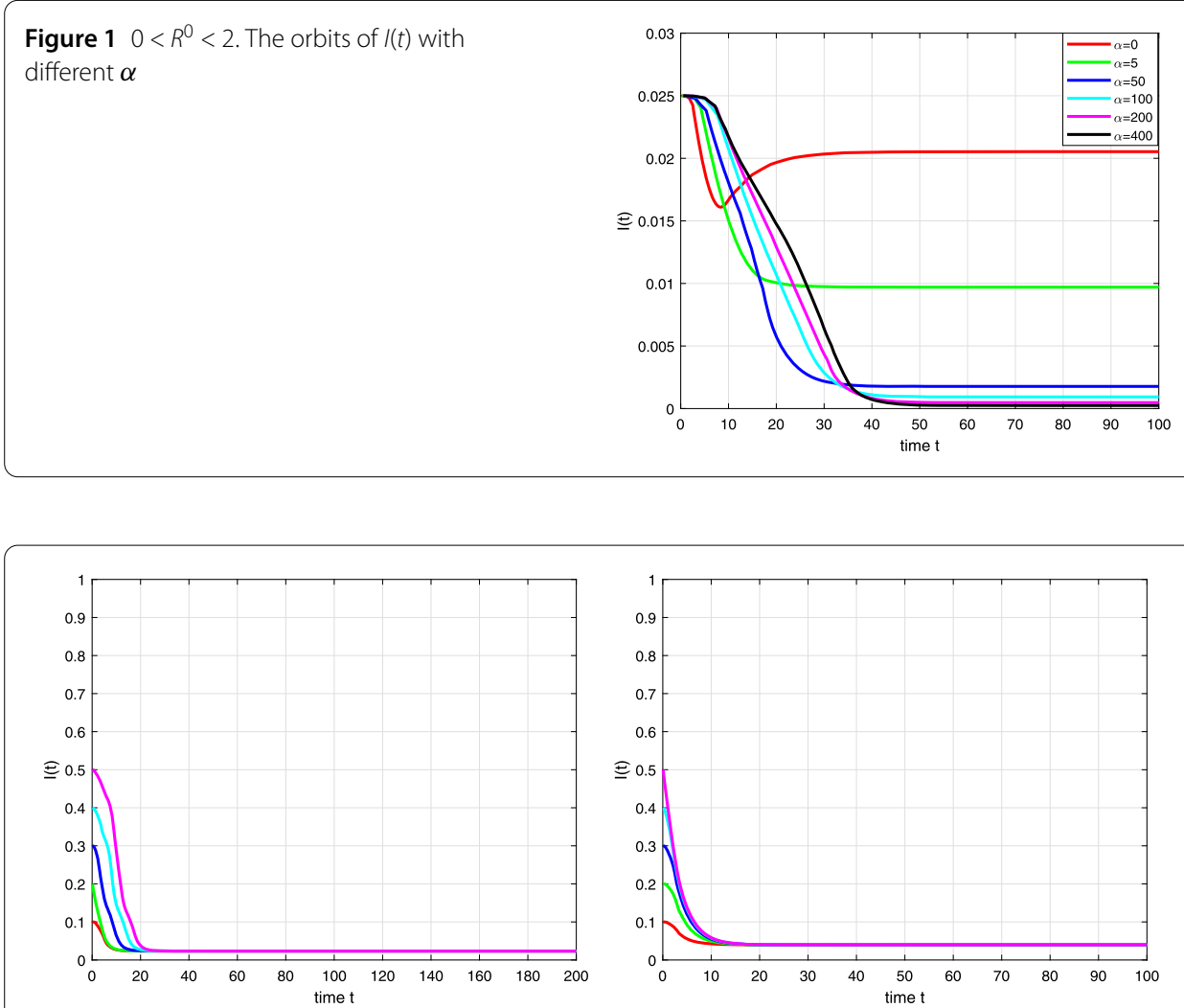

Figure $2 R^{0}>2$. The orbits of $/(t)$ with $\alpha=30$ (left) and $\alpha=15$ (right) 
Figure 3 The relation between $/^{*}$ and $\alpha$

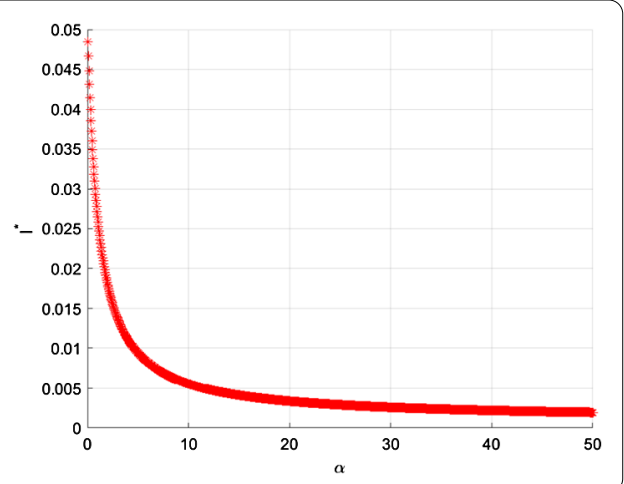

$\alpha$ value in the left figure satisfies the assumption of Theorem 3.1. whereas the $\alpha$ value in the right figure does not, and despite of this, the endemic equilibrium is globally stable.

In Figure 3, the parameters are chosen as follows: $b=0.25, \gamma=0.35$, and $\lambda=0.3$. We can verify that $R^{0}=2.0673>1$. The simulation clearly indicates the relation between $I^{*}$ and $\alpha$, where $I^{*}=\sum_{k=1}^{500} P(k) I_{k}^{*}$.

We can see from the numerical simulations that the when the disease is endemic, the endemic level decreases significantly as $\alpha$ is increased. The biological meaning is that when an epidemic outbreaks, people consciously reduce the number of contacts with others, and the larger the probability one may contact with infected individuals, the more careful the people are, which is consistent with our real experience.

\section{Conclusions}

In this paper, we prove that the disease is permanent if $R^{0}>1$ and that the endemic equilibrium of (1.1) is globally asymptotically stable if $R^{0} \in(1,2]$ and $\alpha>0$, or if $R^{0}>2$ and $\alpha \geq \alpha_{n}=\frac{\lambda n}{4(b+\gamma)}\left(\frac{R^{0}-1}{R^{0}}\right)^{2}$. Moreover, we have performed numerical experiments to illustrate the theoretical results. It is observed from the numerical results that the endemic equilibrium is globally asymptotically stable for any $\alpha>0$ whenever $R^{0}>1$; however, a mathematical proof remains to be very difficult.

Although the feedback parameter $\alpha$ does not affect the threshold $R^{0}$, it plays a role in weakening the spreading of disease, for which a theoretical analysis can be seen from the second inequality of (2.2). Numerical experiments done in [1] and the present paper indicate that the endemic level decreases significantly as the fear factor $\alpha$ increases. The biological meaning is that as soon as an epidemic outbreaks, people are more cautious and reduce contacts with other people consciously, which is beneficial to preventing epidemic spreading.

\section{Appendix}

The following result is due to Barbǎlat [29, Lemma 1.2.3].

Lemma A Let $a \in(-\infty,+\infty)$, and let $f:[a, \infty) \rightarrow \mathbb{R}$ be a differentiable function. If $\lim _{t \rightarrow \infty} f(t)$ exists (finite) and $f^{\prime}(t)$ is uniformly continuous in $(a, \infty)$, then $\lim _{t \rightarrow \infty} f^{\prime}(t)=0$.

The following result is due to Hirsch, Hanisch, and Gabriel [30, Lemma 4.2]; see also [31, Lemma 1.1]. 
Lemma B Suppose that $f: \mathbb{R}^{+} \rightarrow \mathbb{R}$ is a differentiable function. If $\liminf _{t \rightarrow \infty} f<$ $\limsup _{t \rightarrow \infty} f$, then there are two sequences $\left\{\tau_{k}\right\}$ and $\left\{\sigma_{k}\right\}$, increasing to infinity, such that

$$
\begin{array}{ll}
\lim _{k \rightarrow \infty} f\left(\tau_{k}\right)=\limsup _{t \rightarrow \infty} f, & f^{\prime}\left(\tau_{k}\right)=0, \\
\lim _{k \rightarrow \infty} f\left(\sigma_{k}\right)=\liminf _{t \rightarrow \infty} f, & f^{\prime}\left(\sigma_{k}\right)=0 .
\end{array}
$$

The following result is a combination of Lemmas A and B and will be used to show Theorem 2.1.

Corollary C Suppose that $f: \mathbb{R}^{+} \rightarrow \mathbb{R}$ is a bounded twice differentiable function with $f^{\prime \prime}(t)$ bounded in $\mathbb{R}^{+}$. Then there are two sequences $\left\{\tau_{k}\right\}$ and $\left\{\sigma_{k}\right\}$, increasing to infinity, such that

$$
\begin{array}{ll}
\lim _{k \rightarrow \infty} f\left(\tau_{k}\right)=\limsup _{t \rightarrow \infty} f, & \lim _{k \rightarrow \infty} f^{\prime}\left(\tau_{k}\right)=0, \\
\lim _{k \rightarrow \infty} f\left(\sigma_{k}\right)=\liminf _{t \rightarrow \infty} f, & \lim _{k \rightarrow \infty} f^{\prime}\left(\sigma_{k}\right)=0 .
\end{array}
$$

Proof Since $f^{\prime \prime}(t)$ is bounded in $\mathbb{R}^{+}, f^{\prime}(t)$ is uniformly continuous in $\mathbb{R}^{+}$. Consequently, if $\lim _{t \rightarrow \infty} f(t)$ exists, then by Lemma A all previous conclusions hold for any increasing to infinity sequence $\left\{x_{k}\right\}$. Otherwise, $\liminf _{t \rightarrow \infty} f<\limsup _{t \rightarrow \infty} f$. Then the conclusions follow by Lemma B. The proof is completed.

The following result will be used to prove Theorem 4.1.

Lemma $\mathbf{D}$ Let $R^{0}>1$ and $\Theta(0)>0$. Assume that $\left\{\left(S_{k}, I_{k}\right)\right\}_{k=1}^{n}$ is a nonnegative solution of system (1.3) with initial conditions (1.2). Then

(i) If there are constants $u_{k}>0$ for $k=1,2, \ldots, n$ such that

$$
U:=\langle k\rangle^{-1} \sum_{j=1}^{n} j P(j) u_{j} \leq \frac{1}{2 \alpha}, \quad \limsup _{t \rightarrow \infty} I_{k}(t) \leq u_{k}
$$

then

$$
\limsup _{t \rightarrow \infty} I_{k}(t) \leq \frac{\lambda k(1-\alpha U) U}{\gamma+b+\lambda k(1-\alpha U) U} .
$$

(ii) If there are constants $l_{k}>0$ for $k=1,2, \ldots, n$ such that

$$
L:=\langle k\rangle^{-1} \sum_{j=1}^{n} j P(j) l_{j} \leq \frac{1}{2 \alpha}, \quad \liminf _{t \rightarrow \infty} I_{k}(t) \geq l_{k},
$$

then

$$
\liminf _{t \rightarrow \infty} I_{k}(t) \geq \frac{\lambda k(1-\alpha L) L}{\gamma+b+\lambda k(1-\alpha L) L} .
$$

Proof We only prove (i) since (ii) can be proved similarly. 
Indeed, by the same argument as that yielding (2.5) and (2.6) we have

$$
\limsup _{t \rightarrow \infty} I_{k}(t)=\bar{i}_{k}=\frac{\lambda k\left(1-\alpha \bar{\theta}_{k}\right) \bar{\theta}_{k}}{\gamma+b+\lambda k\left(1-\alpha \bar{\theta}_{k}\right) \bar{\theta}_{k}}, \quad k=1,2, \ldots, n .
$$

Note that $0<\bar{\theta}_{k} \leq \lim \sup _{t \rightarrow \infty} \Theta(t) \leq \frac{1}{\langle k\rangle} \sum_{k=1}^{n} k P(k) \lim \sup _{t \rightarrow \infty} I_{k}(t) \leq U \leq \frac{1}{2 \alpha}$. This, together with (4.1), yields (i). Thus, the proof is completed.

\section{Acknowledgements}

We would like to express our deep thanks to referees for their important comments, which greatly improved the paper. We also would like to thank Dr. Lijun Liu for some helpful discussions with him. The research was supported in part by the NSFC (grants 11571062), the Program for Liaoning Excellent Talents in University (grant LU2013124), and the Fundamental Research Fund for the Central Universities (grants DC201502050307, DC201502050202).

\section{Competing interests}

The authors declare that they have no competing interests.

\section{Authors' contributions}

All authors contributed equally to writing this paper. All authors read and approved the final manuscript.

\section{Author details}

${ }^{1}$ College of Computer Science and Technology, Jilin University, Changchun, China. ${ }^{2}$ College of Computer Science, Dalian Nationalities University, Dalian, China. ${ }^{3}$ Department of Mathematics, Dalian Nationalities University, Dalian, China.

\section{Publisher's Note}

Springer Nature remains neutral with regard to jurisdictional claims in published maps and institutional affiliations.

Received: 11 September 2017 Accepted: 19 January 2018 Published online: 17 February 2018

\section{References}

1. Zhang, J., Sun, J.: Stability analysis of an SIS epidemic model with feedback mechanism on networks. Physica A 394, 24-32 (2014)

2. Pastor-Satorras, R., Vespignani, A.: Epidemic spreading in scale-free networks. Phys. Rev. Lett. 86, 3200-3203 (2001)

3. Pastor-Satorras, R., Vespignani, A.: Epidemic dynamics in finite size scale-free networks. Phys. Rev. E 65, Article ID $035108(2002)$

4. Newman, M.E.J.: Spread of epidemic disease on networks. Phys. Rev. E 66, Article ID 016128 (2002)

5. Liu, J., Tang, Y., Yang, Z.: The spreading of disease with birth and death networks. J. Stat. Mech. 2004, Article ID P08008 (2004)

6. Liu, M., Ruan, J: Modelling the spread of sexually transmitted diseases on scale-free networks. Chin. Phys. B 18, 2118-2126 (2009)

7. Liu, J., Zhang, T.: Epidemic spreading of an SEIRS model in scale-free networks. Commun. Nonlinear Sci. Numer. Simul. 16(8), 3375-3384 (2011)

8. Shen, C., Chen, H., Hou, Z:: Strategy to suppress epidemic explosion in heterogeneous metapopulation networks. Phys. Rev. E 86, Article ID 036114 (2012)

9. Zhang, J., Jin, Z:: Epidemic spreading on complex networks with community structure. Appl. Math. Comput. 219(6), 2829-2838 (2012)

10. Ferreira, S., Castellano, S., Pastor-Satorras, R.: Epidemic thresholds of the susceptible-infected-susceptible model on networks: a comparison of numerical and theoretical results. Phys. Rev. E 86, Artical ID 041125 (2012)

11. Li, T., Wang, Y., Guan, Z: Spreading dynamics of a SIQRS epidemic model on scale-free networks. Commun. Nonlinear Sci. Numer. Simul. 19, 686-692 (2014)

12. Fu, X., Small, M., Chen, G.: Propagation Dynamics on Complex Networks: Models, Method and Stability Analysis. Higher Education Press, Beijing (2014)

13. Kiss, I.Z., Miller, J.C., Simon, P.L.: Mathematics of Epidemics on Networks. Springer, Berlin (2017)

14. Wang, L., Dai, G.: Global stability of virus spreading in complex heterogeneous newworks. SIAM J. Appl. Math. 68(5), 1495-1502 (2008)

15. Yang, M., Chen, G., Fu, X.: A modified SIS model with an infective medium on complex networks and its global stability. Physica A 390, 2408-2413 (2011)

16. Zhu, G., Fu, X., Chen, G.: Spreading dynamics and global stability of a generalized epidemic model on complex heterogeneous networks. Appl. Math. Model. 36, 5808-5817 (2012)

17. Wang, Y., Jin, Z., Yang, Z., Zhang, Z., Zhou, T., Sun, G.: Global analysis of an SIS model with an infective vector on complex networks. Nonlinear Anal., Real World Appl. 13, 543-557 (2012)

18. Zhu, G., Fu, X., Chen, G.: Global attractivity of a network-based epidemic SIS model with nonlinear infectivity. Commun. Nonlinear Sci. Numer. Simul. 17, 2588-2594 (2012)

19. Yuan, X., Xue, Y., Liu, M.: Global stability of an SIR model with two susceptible groups on complex networks. Chaos Solitons Fractals 59, 42-50 (2014)

20. Wang, Y., Cao, J.: A note on global stability of the virose equilibrium for network-based computer viruses epidemics. Appl. Math. Comput. 244, 726-740 (2014) 
21. Chen, L., Sun, J: Global stability and optimal control of an SIRS epidemic model on heterogeneous networks. Physica A 410, 196-204 (2014)

22. Chen, L., Sun, J.: Optimal vaccination and treatment of an epidemic network model. Phys. Lett. A 378, 3028-3036 (2014)

23. Li, C., Tsai, C., Yang, S.: Analysis of epidemic spreading of an SIRS model in complex heterogeneous networks. Commun. Nonlinear Sci. Numer. Simul. 19, 1042-1054 (2014)

24. Gong, G., Zhang, D.: An SIS epidemic model with feedback mechanism in scale-free networks. Adv. Mater. Res. 204(210), 354-358 (2011)

25. Li, C.: Dynamics of a network-based SIS epidemic model with nonmonotone incidence rate. Physica A 427, 234-243 (2015)

26. Li, T., Liu, X., Wu, J., Wan, C., Guan, Z., Wang, Y.: An epidemic spreading model on adaptive scale-free networks with feedback mechanism. Physica A 450, 649-656 (2016)

27. D'Onofrio, A.: A note on the global behaviour of the network-based SIS epidemic model. Nonlinear Anal., Real World Appl. 9, 1567-1572 (2008)

28. LaSalle, J.P: The Stability of Dynamical Systems. Regional Conference Series in Applied Mathematics. SIAM, Philadelphia (1976)

29. Golpalsamy, K.: Stability and Oscillations in Delay Differential Equations of Population Dynamics. Kluwer Academic Publishers, Dordrecht (1992)

30. Hirsch, W.M., Hanisch, H., Gabriel, J.-P.: Differential equation model of some parasitic infections: methods for the study of asymptotic behavior. Commun. Pure Appl. Math. 38, 733-753 (1985)

31. Li, B.: Global asymptotic behavior of the chemostat: general response functions and different removal rates. SIAM J. Appl. Math. 59(2), 411-422 (1998)

\section{Submit your manuscript to a SpringerOpen ${ }^{\odot}$ journal and benefit from:}

- Convenient online submission

- Rigorous peer review

- Open access: articles freely available online

High visibility within the field

- Retaining the copyright to your article

Submit your next manuscript at $\boldsymbol{\nabla}$ springeropen.com 